\title{
POLARON EFFECT ON THE BINDING ENERGY OF SHALLOW DONOR IN CYLINDRICAL QUANTUM DOT
}

\author{
R. Charrour, M. Bouhassoune, D. Bria, A. Nougaoui \\ Laboratoire de Dynamique et d'Optique des Matériaux, Faculté des Sciences \\ Université Mohamed $\mathbf{1}^{\mathrm{er}}$, Oujda, Morocco \\ AND M. FLIYOU \\ Equipe de Physique du Solide, ENS, B.P. 5206, Benssouda, Fès, Morocco
}

(Received June 14, 1999; revised version October 26, 1999)

In the framework of the effective-mass approximation and the modified Lee-Low-Pines variational method, we present a theoretical study of the effect of the confined longitudinal-optical phonon and two types of surface-optical phonon (top and side mode) on the binding energy of shallow donor in cylindrical quantum dot. The effect of quantum confinement is described by an infinitely deep potential well. The impact of these different phonon modes is important and depends on the dimension of the quantum dot.

PACS numbers: 71.38.+i, 73.20.Dx, 73.20.Hb

\section{Introduction}

In recent years, a great attention has been devoted to the physics of zero-dimensional nanostructures [1,2]. Interest in the subject of quantum dots is primarily of two kinds: first, the issues related to nanoscales are of utmost importance because of the full quantum nature of the problem and thus have an intrinsic appeal. Second, and more importantly the quantum dot structures have tremendous potentiality of finding applications in microelectronics device technology because of their considerable design flexibility and very many novel physical effects [3-7]. The electron-phonon interaction in these confined systems is one of the important aspects in determining their properties in physical processes, such as the transport phenomena or the electron relaxation phenomena. Roussignal et al. [8] have shown experimentally and explained theoretically that phonon broadening is quite important in very small semiconductor quantum dots. Several studies have already carried out the polarons in quantum box. Gu and co-workers [9-11] have taken into account the interaction of the electron with the confined longitudinal-optical (LO) 
phonons and the surface-optical (SO) phonons in their studies effected in rectangular quantum box. Klimin et al. [12] treated the bulk and interface vibrational modes and their respective contribution to the polaron effects in the nanosphere: the ground-state energy and their effective mass were obtained for a weak coupling, using perturbation theory. Lépine and Bruneau [13] have studied a polaron in the quantum dot: the electron is trapped in an anisotropic parabolic potential and only the coupling to the bulk LO phonon is taken into account. Chen et al. [14] have obtained the interface optical phonon in $\mathrm{GaAs} / \mathrm{Al}_{x} \mathrm{Ga}_{1-x} \mathrm{As}$ as a cylindrical quantum dot (QD) within the framework of the dielectric continuum. Recently, Kanyinda-Malu and de la Cruz [15] have calculated the surface-mode frequencies in the cylindrical dots for both cases: free-standing in vacuum and heterostructure configuration. Following the same treatment as Ref. [16], we have derived a confined LO phonon and two types of SO phonon modes with eigenfrequencies, the Hamiltonian of the free phonon and the electron-phonon interaction Hamiltonian in the dielectric continuum approximation.

The present paper deals with cylindrical quantum dots. The reason of the choice of this geometry lies in the fact that the structures of this type are relatively more convenient to make devices other than those which particularly have spherical geometry. To our knowledge, no theoretical studies of bound polaron in cylindrical quantum dot have yet been reported. Here, we investigate the binding energy of bound polaron to the shallow-doped impurity located at the centre of the dot with infinite potential barriers by means of the Lee-Low-Pines (LLP) variational treatment.

This paper will be organised as follows: In Sec. 2 we present the detailed model based on the variational calculation method. In Sec. 3 we give the numerical results for a GaAs crystal which is widely used in nanodevices domains.

\section{Hamiltonian and formulation}

For the calculations described here, the dot with radius $R$ and height $2 d$ is made of polar crystals and surrounded by the vacuum. The shallow doped impurity is located at the centre of cylindrical quantum dot. To simplify the calculation, we consider that the effective mass approximation remains valid. We also assume that the electrons are confined in an infinitely deep potential well in all directions. The total Hamiltonian

$$
H=H_{\mathrm{e}}+H_{\mathrm{ph}}+H_{\mathrm{e}-\mathrm{ph}},
$$

where

$$
H_{\mathrm{e}}=-\frac{\hbar^{2}}{2 m^{*}}\left(\frac{\partial^{2}}{\partial \rho^{2}}+\frac{1}{\rho} \frac{\partial}{\partial \rho}+\frac{1}{\rho^{2}} \frac{\partial^{2}}{\partial \varphi^{2}}+\frac{\partial^{2}}{\partial z^{2}}\right]-\frac{e^{2}}{\varepsilon_{\infty} r}+V_{\mathrm{c}}(r)
$$

is the Hamiltonian of donor-impurity in the infinite well potential, $m^{*}$ is the electron mass, $\varepsilon_{\infty}$ is the optical dielectric constant, $r=\left(\rho^{2}+z^{2}\right)^{1 / 2}$, and $V_{c}(r)$ is the electron-confining potential well which will be taken as

$$
V_{\mathrm{c}}(r)= \begin{cases}0 & \text { if } \rho \leq R \text { and }|z| \leq d \\ \infty & \text { elsewhere }\end{cases}
$$


While the second term is the total Hamiltonian of the free phonon field in cylindrical $\mathrm{QD}$, i.e.

$$
H_{\mathrm{ph}}=H_{\mathrm{LO}}+H_{\mathrm{TSO}}+H_{\mathrm{SSO}}
$$

where

$$
H_{\mathrm{LO}}=\sum_{l, n_{1}} \hbar \omega_{\mathrm{LO}} a_{l n_{1}}^{+} a_{l n_{1}}
$$

is the Hamiltonian operator for confined LO-phonon, $a_{l_{1}}^{+}\left[a_{l_{n_{1}}}\right]$ are creation [annihilation] operator for the LO-phonon of the $\left(l, n_{1}\right)$-th mode with frequency $\omega_{\text {LO }}$ (they satisfy. the commutative rules for bosons) and wave vector $\left(k_{\|}=\chi_{n_{1}} / R\right.$, $\left.k_{z}=l \pi / 2 d\right)$ where $\chi_{n_{1}}$ is the $n_{1}$-th root of the Bessel function of the zero order.

The Hamiltonian operators for top SO (TSO) mode and side SO (SSO) mode phonons are respectively

$$
H_{\mathrm{TSO}}=\sum_{n_{2}, m, p} \hbar \omega_{p} b_{n_{2}, m, p}^{+} b_{n_{2}, m, p}
$$

and

$$
H_{\mathrm{SSO}}=\sum_{n_{3}, m, p} \hbar \omega_{s s} B_{n_{3}, m, p}^{+} B_{n_{3}, m, p}
$$

where $b_{n_{2}, m, \pm}^{+} b_{n_{2}, m, \pm}$ and $B_{n_{3}, m, \pm}^{+} B_{n_{3}, m, \pm}$ are, respectively, the creation and annihilation operators of TSO and SSO phonons with frequency $\omega_{ \pm}$and $\omega_{s s}$ of the $\left(n_{2}, m\right)$-th and $\left(n_{3}, m\right)$-th modes. $p=+;-$ for symmetric and antisymmetric mode respectively.

For surface phonons, the intersubband transition can be neglected $[17,18]$ and the electrons are assumed to occupy only the ground-subband $(n=1, m=0)$. Therefore, we consider only the lowest surface phonon modes $(m=0)$.

By using the standard boundary conditions of electrostatic and taking into account the geometry of cylindrical QD, we obtained the eigenfrequencies for different modes

$$
\begin{aligned}
& \omega_{\mathrm{LO}}^{2}=\frac{\varepsilon_{0}}{\varepsilon_{\infty}} \omega_{\text {TO }}^{2}, \\
& \omega_{\mathrm{TSO} \pm}^{2}=\frac{\left(\varepsilon_{0}+1\right) \mp\left(\varepsilon_{0}-1\right) \exp \left(-2 q_{ \pm} d\right)}{\left(\varepsilon_{\infty}+1\right) \mp\left(\varepsilon_{\infty}-1\right) \exp \left(-2 q_{ \pm} d\right)} \omega_{\text {TO }}^{2}, \\
& \omega_{\mathrm{SSO}}^{2}=\left(1-\frac{\varepsilon_{0}+\varepsilon_{\infty}}{\varepsilon_{\infty}-\varepsilon}\right) \omega_{\mathrm{TO}}^{2},
\end{aligned}
$$

where

$$
\varepsilon=-\frac{I_{0}\left(k_{n_{3}} R\right) K_{1}\left(k_{n_{3}} R\right)}{I_{1}\left(k_{n_{3}} R\right) K_{0}\left(k_{n_{3}} R\right)} .
$$

$I_{0}, K_{0}, I_{1}, K_{1}$ are the modified Bessel functions of zero and one order. $k_{n_{3}}=n_{3} \pi / 2 d$ is the wave vector of the SSO phonon modes.

The corresponding TSO wave vectors $\left(q_{n_{2}, \pm}\right)$ are the $n_{2}$-th root of equations

$$
\pi q_{n_{2},+} R J_{1}\left(q_{n_{2},+} R\right) J_{0}\left(q_{n_{2},+} R\right)+\exp \left(-2 q_{n_{2},+} d\right)-1=0 \text {, }
$$




$$
\pi q_{n_{2},-} R J_{1}\left(q_{n_{2},-} R\right) J_{0}\left(q_{n_{2},-} R\right)-\exp \left(-2 q_{n_{2},-} d\right)-1=0
$$

the number of wave vectors $\left(q_{n_{2}, \pm}\right)$ is limited by the Brillouin zone, i.e. $q_{n_{2}, \pm} \leq \pi / 2 a$, where $a$ is the lattice constant of the crystal.

The last term in Eq. (1) describes the Hamiltonian interaction of an electron with different phonon modes

$$
H_{\text {int }}=H_{\mathrm{e}-\mathrm{LO}}+H_{\mathrm{e}-\mathrm{TsO}}+H_{\mathrm{e}-\mathrm{SsO}} \text {. }
$$

The first term is the electron-LO-phonon interaction

$$
\begin{aligned}
H_{\mathrm{e}-\mathrm{LO}} & =-\sum_{n_{1}} J_{0}\left(\chi_{n_{1}} \frac{\rho}{R}\right)\left[\sum_{l=1,3 \ldots} V_{l n_{1}} \cos \left(\frac{l \pi z}{2 d}\right)\left(a_{l n_{1}}+a_{l n_{1}}^{+}\right)\right. \\
& \left.+\sum_{l=2,4 \ldots} V_{l n_{1}} \sin \left(\frac{l \pi z}{2 d}\right)\left(a_{l n_{1}}+a_{l n_{1}}^{+}\right)\right]
\end{aligned}
$$

with

$$
V_{l n_{1}}^{2}=\frac{1}{\Omega} \frac{4 \pi e^{2} \hbar \omega_{L O}}{\left(\frac{\chi_{n_{1}}}{R}\right)^{2} J_{1}^{2}\left(\chi_{n_{1}}\right)\left[1+\left(\frac{l \pi R}{2 d \chi_{n_{1}}}\right)^{2}\right]}\left(\frac{1}{\varepsilon_{\infty}}-\frac{1}{\epsilon_{0}}\right),
$$

where $\Omega=2 \pi R^{2} d$ is the crystal volume.

The second term is the electron-TSO-phonon interaction, which is given by

$$
\begin{gathered}
H_{\mathrm{e}-\mathrm{TSO}}=-\sum_{n_{2},+} V_{n_{2},+} J_{0}\left(q_{n_{2},+} \rho\right) \cosh \left(q_{n_{2},+} z\right)\left(b_{n_{2},+}+b_{n_{2},+}^{+}\right) \\
-\sum_{n_{2},-} V_{n_{2},-} J_{0}\left(q_{n_{2},-} \rho\right) \sinh \left(q_{n_{2},-} z\right)\left(b_{n_{2},-}+b_{n_{2},-}^{+}\right)
\end{gathered}
$$

with

and

$$
\begin{aligned}
& V_{n_{2,+}}^{2}=\frac{4 \pi e^{2} \hbar \omega_{+}}{S q_{n_{2},+}} \\
& \times \frac{\frac{1}{\varepsilon\left(\omega_{+}\right)-\varepsilon_{0}}-\frac{1}{\varepsilon\left(\omega_{+}\right)-\varepsilon_{\infty}}}{\left[\begin{array}{l}
\left(\sinh \left(2 q_{n_{2}}, d\right)+2 q_{n_{2},+} d\right)\left(J_{1}^{2}\left(q_{n_{2},+} R\right)-J_{0}\left(q_{n_{2},}, R\right) J_{2}\left(q_{n_{2},} R\right)\right) \\
+\left(\sinh \left(2 q_{n_{2}}, d\right)-2 q_{n_{2}},+d\right)\left(J_{0}^{2}\left(q_{n_{2},}+R\right)+J_{1}^{2}\left(q_{n_{2}}, R\right)\right)
\end{array}\right]}
\end{aligned}
$$

$$
\begin{aligned}
& V_{n_{2,-}}^{2}=\frac{4 \pi e^{2} \hbar \omega_{-}}{S q_{n_{2},-}}
\end{aligned}
$$

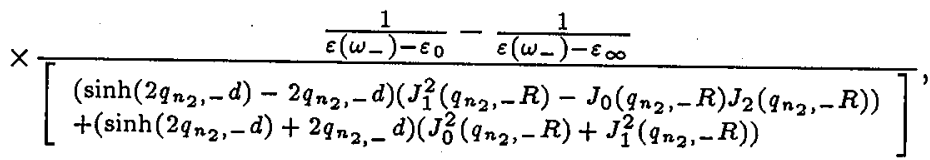

where

$$
\varepsilon\left(\omega_{+}\right)=-\operatorname{coth}\left(q_{n_{2},+} d\right)
$$

and

$$
\varepsilon\left(\omega_{-}\right)=-\tanh \left(q_{n_{2},-} d\right) .
$$


The last term in Eq. (13) is the electron-SSO-phonon interaction

$$
\begin{gathered}
H_{\mathrm{e}-\mathrm{SSO}}=-\sum_{n_{3}=2,4 \ldots} \Gamma_{n_{3},+} I_{0}\left(\frac{n_{3} \pi}{2 d} \rho\right) \cos \left(\frac{n_{3} \pi}{2 d} z\right)\left(B_{n_{3},+}+B_{n_{3},+}^{+}\right) \\
-\sum_{n_{3}=1,3 \ldots} \Gamma_{n_{3},-} I_{0}\left(\frac{n_{3} \pi}{2 d} \rho\right) \sin \left(\frac{n_{3} \pi}{2 d} z\right)\left(B_{n_{3},-}+B_{n_{3},-}^{+}\right)
\end{gathered}
$$

with

$$
\begin{aligned}
\Gamma_{n_{3}, \pm}^{2} & =\frac{1}{S} \frac{2 \pi e^{2} \hbar \omega_{s s}}{d k_{n_{3}}^{2}\left[I_{0}^{2}\left(k_{n_{3}} R\right)-I_{0}\left(k_{n_{3}} R\right) I_{2}\left(k_{n_{3}} R\right)\right]} \\
\times & {\left[\frac{1}{\varepsilon\left(\omega_{s s}\right)-\varepsilon_{0}}-\frac{1}{\varepsilon\left(\omega_{s s}\right)-\varepsilon_{\infty}}\right], }
\end{aligned}
$$

where $S=\pi R^{2}$ is the cross-sectional (perpendicular to $Z$ ) area of the cylindrical dot.

In the following we shall adopt the variational treatment developed by Lee et al. [19] to deal with the Hamiltonian (1). To facilitate the deductions, we use the following unitary transformation:

$$
\begin{aligned}
U= & \exp \left[\sum_{\ln _{1}}\left(f_{l n_{1}} a_{l_{1}}^{+}-\text {c.c. }\right)+\sum_{n_{2}, p}\left(g_{n_{2}, p} b_{n_{2}, p}^{+}-\text {c.c. }\right)\right. \\
& \left.+\sum_{n_{3, p}}\left(h_{n_{3}, p} B_{n_{3}, p}^{+}-\text {c.c. }\right)\right],
\end{aligned}
$$

where $f_{l_{n_{1}}}, g_{n_{2}, p}$ and $h_{n_{3}, p}$ are the variational parameters which will subsequently be selected by minimising the energy of the system.

At low temperature limit, the wave function of the system can be written as$$
|\psi(\rho, z)\rangle=\left|\phi_{\mathrm{e}}(\rho, z)\right\rangle|0\rangle,
$$

where $\phi_{\mathrm{e}}(\rho, z)$ is the wave function of the electron moving in the dot and $|0\rangle$ is the vacuum state of the phonon. The expectation value of the Hamiltonian $H$ in such a state is given by

$$
E=\left\langle\psi(\rho, z)\left|U^{-1} H U\right| \psi(\rho, z)\right\rangle=\left\langle\phi_{\mathrm{e}}(\rho, z)|F| \phi_{\mathrm{e}}(\rho, z)\right\rangle,
$$

where

$$
F=\left\langle 0\left|U^{-1} H U\right| 0\right\rangle=F\left(\rho, z, f_{l_{1}}, f_{l n_{1}}^{+}, g_{n_{2}, p}, g_{n_{2}, p}^{+}, h_{n_{3}, p}, h_{n_{3}, p}^{+}\right) .
$$

The variational conditions $\partial F / \partial f_{l_{n_{1}}}=0, \partial F / \partial g_{n_{2}, p}=0$, and $\partial F / \partial h_{n_{3}, p}=0$ can be used to determine the forms of $f_{l_{n_{1}}}, g_{n_{2}, p}$, and $h_{n_{3}, p}$, which are

$$
\begin{aligned}
& f_{l n_{1}}=\frac{V_{l n_{1}}}{\hbar \omega_{\mathrm{LO}}} J_{0}\left(\chi_{n_{1}} \frac{\rho}{R}\right) \operatorname{csn}\left(\frac{l \pi}{2 d} z\right), \\
& g_{n_{2},+}=\frac{V_{n_{2},+}}{\hbar \omega_{+}} J_{0}\left(q_{n_{2},+} \rho\right) \cosh \left(q_{n_{2},+} z\right), \\
& g_{n_{2},-}=\frac{V_{n_{2},-}}{\hbar \omega_{-}} J_{0}\left(q_{n_{2},-} \rho\right) \sinh \left(q_{n_{2},-} z\right),
\end{aligned}
$$




$$
h_{n_{3}, p}=\frac{\Gamma_{n_{3}, p}}{\hbar \omega_{s s}} I_{0}\left(\frac{n_{3} \pi}{2 d} \rho\right) \operatorname{scn}\left(\frac{n_{3} \pi}{2 d} z\right),
$$

where, for convenience, the functions $\operatorname{csn}\left(\frac{l \pi}{2 d} z\right)$ and $\operatorname{sen}\left(\frac{n_{3} \pi}{2 d} z\right)$ are defined as

$$
\begin{aligned}
& \operatorname{csn}\left(\frac{l \pi}{2 d} z\right)= \begin{cases}\cos \left(\frac{l \pi}{2 d} z\right) & \text { for odd } l, \\
\sin \left(\frac{l \pi}{2 d} z\right) & \text { for even } l,\end{cases} \\
& \operatorname{sen}\left(\frac{n_{3} \pi}{2 d} z\right)= \begin{cases}\cos \left(\frac{n_{3} \pi}{2 d} z\right) & \text { for even } n_{3}, \\
\sin \left(\frac{n_{3} \pi}{2 d} z\right) & \text { for odd } n_{3} .\end{cases}
\end{aligned}
$$

We take the variational minimum of $F$ as the effective Hamiltonian of the bound polaron in a quantum dot

$$
\begin{aligned}
H_{\mathrm{eff}} & =\min \{F\}=-\frac{\hbar^{2}}{2 m^{*}}\left(\frac{\partial^{2}}{\partial \rho^{2}}+\frac{1}{\rho} \frac{\partial}{\partial \rho}+\frac{1}{\rho^{2}} \frac{\partial^{2}}{\partial \varphi^{2}}+\frac{\partial^{2}}{\partial z^{2}}\right) \\
& -\frac{e^{2}}{\varepsilon_{\infty} r}+V_{\mathrm{c}}(r)+V_{\mathrm{LO}}(\rho, z)+V_{\mathrm{TSO}}(\rho, z)+V_{\mathrm{SSO}}(\rho, z) .
\end{aligned}
$$

$V_{\mathrm{LO}}(\rho, z), V_{\mathrm{TSO}}(\rho, z)$, and $V_{\mathrm{SSO}}(\rho, z)$ are the effective potentials induced by the interaction between the electron with the confined LO-phonon, the top-SO-phonon and the side-SO-phonon, respectively

$$
\begin{aligned}
& V_{\mathrm{LO}}(\rho, z)=-\sum_{n_{1}} \frac{J_{0}^{2}\left(\frac{\chi_{n_{1}}}{R} \rho\right)}{\hbar \omega_{\mathrm{LO}}} \\
& \times\left[\sum_{l=1,3} V_{l_{n_{1}}}^{2} \cos ^{2}\left(\frac{l \pi}{2 d} z\right)+\sum_{1=2,4} V_{l_{n_{1}}}^{2} \sin ^{2}\left(\frac{l \pi}{2 d} z\right)\right], \\
& V_{\mathrm{TSO}}(\rho, z)=-\sum_{n_{2},+} \frac{V_{n_{2},+}^{2}}{\hbar \omega_{+}} J_{0}^{2}\left(q_{n_{2},+} \rho\right) \cosh ^{2}\left(q_{n_{3},+} z\right) \\
& \quad-\sum_{n_{2},-} \frac{V_{n_{2},-}^{2}}{\hbar \omega_{-}} J_{0}^{2}\left(q_{n_{2},-} \rho\right) \sinh ^{2}\left(q_{n_{2},-} z\right), \\
& V_{\mathrm{SSO}}(\rho, z)=-\sum_{n_{3}=2,4} \frac{\Gamma_{n_{3},+}^{2}}{\hbar \omega_{s s}} I_{0}^{2}\left(n_{3} \frac{\pi \rho}{2 d}\right) \cos ^{2}\left(n_{3} \frac{\pi z}{2 d}\right) \\
& \quad-\sum_{n_{3}=1,3} \frac{\Gamma_{n_{3},-}^{2}}{\hbar \omega_{s s}} I_{0}^{2}\left(n_{3} \frac{\pi \rho}{2 d}\right) \sin ^{2}\left(n_{3} \frac{\pi z}{2 d}\right) .
\end{aligned}
$$

In the following, we use the atomic units $a^{*}=\hbar^{2} \varepsilon_{\infty} /\left(m^{*} e^{2}\right)$ for length and $R^{*}=m^{*} e^{4} /\left(2 \varepsilon_{\infty}^{2} \hbar^{2}\right)$ for energy. In these units, the effective Hamiltonian becomes

$$
\begin{aligned}
H_{\mathrm{eff}} & =-\left(\frac{\partial^{2}}{\partial \rho^{2}}+\frac{1}{\rho} \frac{\partial}{\partial \rho}+\frac{1}{\rho^{2}} \frac{\partial^{2}}{\partial \varphi^{2}}+\frac{\partial^{2}}{\partial z^{2}}\right)-\frac{2}{\sqrt{\rho^{2}+z^{2}}} \\
& +V_{\mathrm{c}}(r)+V_{\mathrm{LO}}(\rho, z)+V_{\mathrm{TSO}}(\rho, z)+V_{\mathrm{SSO}}(\rho, z) .
\end{aligned}
$$


In order to calculate the impurity binding energy for the ground state electron subband we consider the following ground state wave function:

$$
\phi_{\mathrm{e}}(\rho, z)=N J_{0}\left(\frac{\theta_{0}}{R} \rho\right) \cos \left(\frac{\pi z}{2 d}\right) \exp (-\alpha \rho),
$$

where $J_{0}, N, \alpha$, and $\theta_{0}$ are, respectively, the Bessel function of zero order, the normalisation constant, the variational parameter, and the first root of $J_{0}(x)$. The first factor in Eq. (34) describes the confined electron [20] while the exponential factor accounts for the correlation effect between the electron and the positive charge localised at $(\rho=0, z=0)$. Such a separable form in $\rho$ and $z$ of the wave function is valid only for $R \geq H$. For $R \ll H$ a more convenient form of the wave function should contain $\exp \left[-\alpha\left(\rho^{2}+z^{2}\right)^{1 / 2}\right]$ factor accounting for the presence of a donor potential. The ground state energy obtained is

$$
E=\left\langle\phi_{\mathrm{e}}(\rho, z)\left|H_{\mathrm{eff}}\right| \phi_{\mathrm{e}}(\rho, z)\right\rangle=E_{0}+E_{\mathrm{LO}}+E_{\mathrm{TSO}}+E_{\mathrm{SSO}},
$$

where

$$
\begin{aligned}
E_{0} & =\left\langle\phi_{\mathrm{e}}(\rho, z)\left|-\nabla_{r}^{2}-\frac{2}{r}+V_{\mathrm{c}}(r)\right| \phi_{\mathrm{e}}(\rho, z)\right\rangle \\
& =\left(\frac{\pi}{2 d}\right)^{2}+\left(\frac{\theta_{0}}{R}\right)^{2}-\alpha^{2}+\frac{\alpha P_{2}}{R P_{1}}-\frac{2 \alpha \theta_{0} P_{3}}{R P_{1}}-\frac{2}{d} \frac{P_{4}}{P_{1}} .
\end{aligned}
$$

The integrals $P_{i}$ are defined by

$$
\begin{aligned}
& P_{1}=\int_{0}^{1} x J_{0}^{2}\left(\theta_{0} x\right) \exp (-2 R \alpha x) \mathrm{d} x \\
& P_{2}=\int_{0}^{1} J_{0}^{2}\left(\theta_{0} x\right) \exp (-2 R \alpha x) \mathrm{d} x, \\
& P_{3}=\int_{0}^{1} x J_{0}\left(\theta_{0} x\right) J_{1}\left(\theta_{0} x\right) \exp (-2 R \alpha x) \mathrm{d} x \\
& P_{4}=\int_{0}^{1} x J_{0}^{2}\left(\theta_{0} x\right) \exp (-2 R \alpha x) \int_{-d}^{d} \frac{\cos ^{2}(\pi z / 2 d)}{\sqrt{(R x)^{2}+z^{2}}} \mathrm{~d} z \mathrm{~d} x .
\end{aligned}
$$

$E_{\mathrm{LO}}, E_{\mathrm{TSO}}$, and $E_{\mathrm{SSO}}$ are the contributions of different phonon modes to the ground state energy of bound polaron in cylindrical quantum box. Their expressions are so long that we cannot write them here.

The impurity binding energy $E_{\mathrm{B}}$ is defined as the difference of energy between the bottom of the electronic conduction band without the impurity $E_{\text {sub }}$ and the ground level of the impurity in the quantum dot

$$
E_{\mathrm{B}}=E_{\mathrm{sub}}-\min _{\alpha}\left\langle\phi_{\mathrm{e}}(r)\left|H_{\mathrm{eff}}\right| \phi_{\mathrm{e}}(r)\right\rangle \text {. }
$$

\section{Numerical results}

This section deals with numerical illustrations of our theoretical results on the polar crystal of GaAs which has the corresponding physical parameters [21]: 
$m^{*}=0.0657 m_{0}, \hbar \omega_{\mathrm{LO}}=36.7 \mathrm{meV}, \varepsilon_{0}=12.8$ and $\varepsilon_{\infty}=10.9$, where $m_{0}$ is the free-electron mass.

Equation (13) represents the Hamiltonian interaction of an electron with three different types of phonon modes. We will be interested in the evolution of these contributions as function of the system dimensions and in their effects on the binding energy of shallow donor in quantum dot. In Fig. 1 we have reported the variation of the binding energy against $R$ for different values of the height box $2 d$ in the presence and absence of the LO-phonon modes. It appears, as expected, that the interaction with the LO-phonon increases the binding energy and it depends on the height of cylindrical quantum box. For a fixed large radius, we can see that this latter energy varies between $4 R^{*}$ (quantum disk limit [20]) and $1 R^{*}$ (bulk limit) according to the height value. In addition, the correction $\Delta E_{\text {LO }}$ (Fig. 2) decreases rapidly as $R$ increases and it is more important in the quantum dot of thin width. Since the longitudinal-optical phonons propagated along the $z$-axis, the LO-phonon number increases with the box height. Besides, the lateral confinement augments the phonon density; therefore, their interaction with an electron will be important. The values of the height $H$ considered here are small compared with those of the radius $R$. For larger radius $R \geq H$ the form of the wave function Eq. (34) is valid. However for small values of $R$, the binding energy will reach a maximum and decrease after for a finite potential barrier. In this region we know
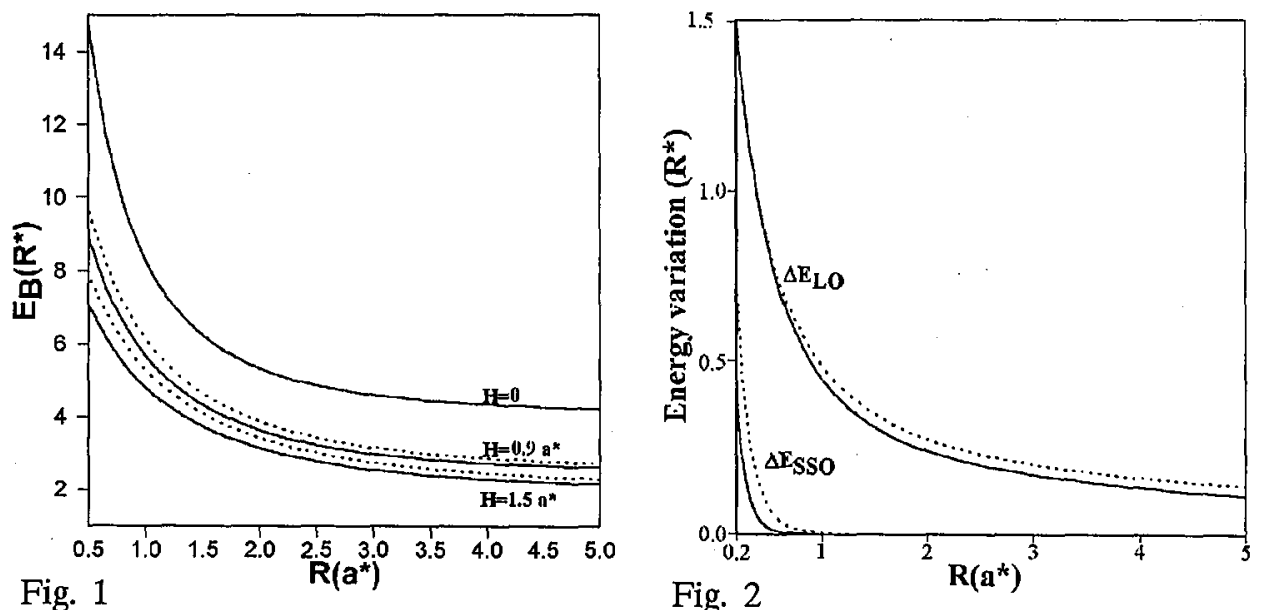

Fig. 1. Binding energy $E_{B}$ of shallow-doped impurity located at the centre of the GaAs quantum cylinder with (dotted line) and without (solid line) electron-LO-phonon coupling plotted against $R$ for $H=0$ (disk limit) and two other values of the height $(H=2 d)$.

Fig. 2. Polaronic corrections ( $\Delta E_{\mathrm{LO}}$ and $\Delta E_{\mathrm{SSO}}$ ) to the binding energy of the bound electron in GaAs QD as function of the cylindrical radius $R$ for two values of the QD height $H=2 d=1.54$ (dotted line) and $H=2 d=0.9$ (solid line). 
that the result obtained here overestimated the binding energy and the wave function will be written as $\exp \left[-\alpha\left(\rho^{2}+z^{2}\right)^{1 / 2}\right]$.

The effect of the correction, due to the SSO phonon, on the binding energy is significant only for a dot characterised by small radius $R$. This correction $\Delta E_{\text {Sso }}$ (Fig. 2) decreases rapidly as the width of the cylindrical QD increases, and augments according to the increase in height $2 d$. Indeed, the bound electron to the impurity, placed at the centre of QD, feels better the effect of SSO phonon modes when it is close to lateral surface (small width $R$ ) $[22,23]$.

On the strength of the above curve (Fig. 2) we notice that on the one hand, both corrections augment proportionally with the height and on the other hand the $\Delta E_{\mathrm{SSO}}$ correction is subjected to a strong attenuation in comparison with $\Delta E_{\mathrm{LO}}$. The same results are shown for the surface optical phonon modes in spherical quantum dot [24].

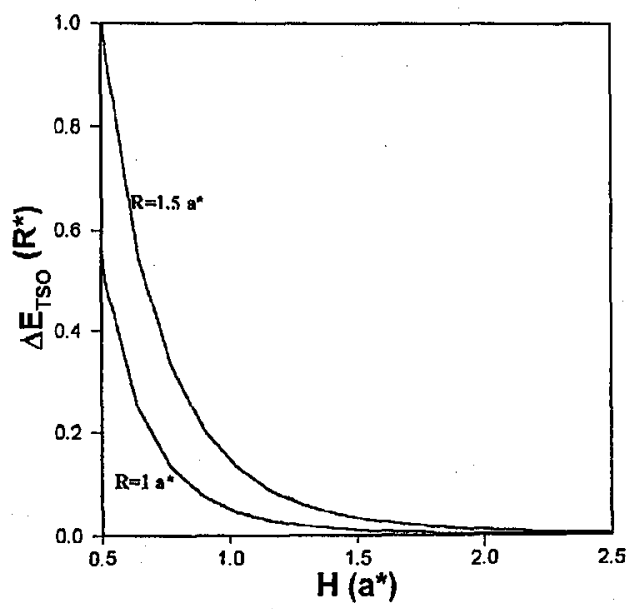

Fig. 3. Polaronic correction ( $\left.\Delta E_{\mathrm{TSO}}\right)$ to the bound electron in GaAs $\mathrm{QD}$ as a function of the QD height $(H=2 d)$ for two values of the cylindrical radius $R$.

The effect of TSO phonon modes on the binding energy presents a prominent impact only for the small dot (weak thickness). We observe in Fig. 3 that the contribution of this type of surface phonons $\left(\Delta E_{\mathrm{TSO}}\right)$ declines rapidly with the augmentation of the QD thickness and vanishes in the big values of the QD height (quantum well wire limit).

As for fixed small values of the height $2 d(2 d<1)$, the correction $\Delta E_{\text {TsO }}$ increases with the increase in radius until reaching the quantum well limit [21].

The aim of this work is not to give the quantitative values of the binding energy but only the formalism and its application to an ideal case where infinite barrier height and a single variational parameter wave function $\exp (-\alpha \rho)$ are taken into account. A study of more realistic case with finite potential barrier and an appropriate wave function is in progress. 


\section{Conclusion}

With the use of the variational method to solve the effective mass equation, we have studied two effects on the binding energy of the shallow-doped impurity located at the centre of a cylindrical dot: the polaronic effect and implicitly the dot size effect. We have shown that the polaronic corrections increase as the confinement size (height and width) decreases. Indeed, for small cylinder dimensions, the impact of these different phonon modes is very important and must be taken into account.

\section{Acknowledgment}

This work was supported by the programs-in-aide for Scientific Research (PARS), Physique 16 Oujda and Physique 03 Fès. The authors wish to thank Mr. B. Stébé for his helpful discussions.

\section{References}

[1] A.D. Yoffe, Adv. Phys. 42, 173 (1993).

[2] U. Woggon, S.V. Gaponenko, Phys. Status Solidi B 189, 285 (1995).

[3] T. Demel, D. Heihnann, P. Grambow, K. Ploog, Phys. 'Rev. Lett, 64, 788 (1990).

[4] A. Lorke, J.P. Kotthauss, K. Ploog, Phys. Rev. Lett. 64, 2559 (1990).

[5] C.T. Liu, K. Nakamura, D.C. Tsui, K. Ismail, D.A. Antoniadis, H.I. Smith, Appl. Phys. Lett. 55, 168 (1989).

[6] W. Hansen, T.P. Smith III, K.Y. Lee, J.A. Brum, C.M. Knoedler, J.M. Hong, D.P. Kern, Phys. Rev. Lett. 62, 2168 (1989).

[7] M. Tewordt, V. Law, M. Kelly, R. Newburg, M. Pepper, D. Peacock, J. Frost, D. Ritchie, G. Jone, J. Phys., Condens. Matter 2, 8969 (1990).

[8] P. Roussignal, D. Ricard, C. Flytzanis, Phys. Rev. Lett. 62, 312 (1989).

[9] H.Y. Zhou, Shi Wei Gu, Solid State Commun. 86, 403 (1993).

[10] Ka-Di. Zhu, Shi Wei Gu, Solid State Commun. 85, 651 (1993).

[11] Ka-Di. Zhu, Shi Wei Gu, Solid State Commun. 81, 211 (1992).

[12] S.N. Klimin, E.P. Pokatilov, V.M. Fomin, Phys. Status Solidi B 184, 373 (1994).

[13] Y. Lépine, G. Bruneau, J. Phys., Condens. Matter 10, 1495 (1998).

[14] Chuan-yu chen, Wai-sang Li, Ho-Kee Yeung, Solid State Commun. 106, 341 (1998).

[15] C. Kanyinda-Malu, R.M. de la Cruz, Phys. Rev. B 59, 1621 (1999).

[16] Wai-sang Li, Chuan-yu chen, Physica B 229, 375 (1997).

[17] N.C. Constantinou, B.K. Ridley, Phys. Rev. B 41, 10622 (1990).

[18] X.F. Wang, X.L. Lei, Phys. Rev. B 49, 4780 (1994).

[19] T.D. Lee, F.E. Low, D. Pines, Phys. Rev. 90, 297 (1953).

[20] S. Le Goff, B. Stebe, Phys. Rev. B 47, 1383 (1993).

[21] Y. Chen Li, L.F. Zheng, S.W. Gu, Phys. Rev. B 38, 4096 (1988).

[22] H.Y. Zhou, S.W. Gu, Solid State Commun. 88, 291 (1993).

[23] W.D. Sheng, Y.O. Xiao, S.W. Gu, J. Phys., Condens. Matter 5, 1129 (1993).

[24] M. Fliyou, H. Satori, M. Bonayad, Phys. Status Solidi B 212, 97 (1999). 